\title{
Les suites du choc PISA dans les médias allemands
}

The aftermath of the PISA shock in the German media

Las consecuencias de la conmoción PISA en los medios de comunicación alemanes

\section{Jeanne Rubner}

Traducteur : Sylvaine Herold et Marie-José Sanselme

\section{OpenEdition}

\section{Journals}

Édition électronique

URL : http://journals.openedition.org/ries/4009

DOI : 10.4000/ries.4009

ISSN : 2261-4265

Éditeur

Centre international d'études pédagogiques

Édition imprimée

Date de publication : 1 septembre 2014

Pagination : 99-107

ISBN : 978-2-85420-604-3

ISSN : $1254-4590$

\section{Référence électronique}

Jeanne Rubner, "Les suites du choc PISA dans les médias allemands », Revue internationale

d'éducation de Sèvres [En ligne], 66 | septembre 2014, mis en ligne le 01 septembre 2016, consulté le

22 juin 2020. URL : http://journals.openedition.org/ries/4009 ; DOI : https://doi.org/10.4000/ries.4009 


\section{Les suites du choc PISA dans les médias allemands*}

\section{Jeanne Rubner}

\section{SEIZE LÄNDER, SEIZE SYSTÈMES SCOLAIRES}

Nous sommes toujours marqués par l'Histoire. Lorsque les Alliés, après la Seconde Guerre mondiale, créèrent un nouvel ordre politique pour l'Allemagne de l'Ouest, ils veillèrent à ce qu'aucun État centralisé ne puisse voir le jour. L'éducation devint également une compétence des Länder. Un Jules Ferry n'aurait pu, en République fédérale allemande, donner à l'école un visage républicain.

Au contraire, le ministre de l'éducation et de la culture ${ }^{1}$ de chaque Land, ou plutôt le parti au pouvoir, décide quelles écoles sont bonnes pour les enfants de chaque Land. Avec des conséquences importantes : dans certaines régions, comme la Rhénanie du Nord-Westphalie, des établissements polyvalents ${ }^{2}$ ont vu le jour, faisant office d'établissements secondaires uniques pour tous les enfants jusqu'à la dixième année ; dans d'autres États, comme la Bavière, il existe trois filières scolaires parallèles - la Hauptschule (comprenant un cycle primaire allongé, jusqu'à la neuvième classe), la Realschule, qui permet une poursuite d'études dans la voie professionnelle, et le Gymnasium, ou lycée. Il en résulte que le débat sur ce que devrait apporter l'école tourne presque toujours, en pratique, autour de cette question de l'école unique vs système scolaire tripartite ; d'une école fournissant le plus grand soutien possible à tous les enfants du même niveau (défendue par les sociaux-démocrates) vs une école orientée en fonction des capacités et de la réussite (portée par les partis conservateurs). Les onze ministres de l'éducation et de la culture, devenus seize depuis la réunification de l'Allemagne, se battent sur cette question. Encore aujourd'hui, au sein de la Conférence permanente des ministres de l'Éducation et de la Culture, qui réunit l'ensemble des Länder, deux groupes se distinguent: le groupe A, des Länder gouvernés par le SPD, le parti social-démocrate et le groupe B, des Länder gouvernés par la CDU, le parti démocrate-chrétien ou la CSU, l’Union chrétiennesociale, des partis conservateurs ${ }^{3}$.

\footnotetext{
* Article traduit par Sylvaine Herold, avec Marie-José Sanselme.

1. Kultusminister en allemand $(N d T)$.

2. Gesamtschule en allemand : fait référence à un établissement scolaire de niveau secondaire regroupant les trois filières (Hauptschule, Realschule, Gymnasium) (NdT).

3. Pour en savoir plus sur l'histoire de la conférence des ministres de l'éducation de 1948 à 1998 : www.kmk.org/ wir-ueber-uns/gruendung.../zur-geschichte-der-kmk.html.
} 
Ce que les enfants apprennent ne dépend donc pas seulement de l'école qu'ils fréquentent, mais de l'endroit où ils habitent. À chaque type d'école s'applique la formule: un Land, un programme scolaire. En d'autres termes : seize Länder, seize programmes scolaires (si la République démocratique allemande avait un système scolaire centralisé, les cinq "nouveaux » Länder de l'Est se sont cependant très vite adaptés à la juxtaposition fédérale de l'Ouest). Cela devient un véritable casse-tête pour les parents, si leur enfant n'a pas appris l'analyse combinatoire ou le latin en Basse-Saxe, par exemple, et doit aller à l'école en Bavière en cas de déménagement. Les programmes scolaires sont également établis en fonction de considérations politiques : dans les Länder conservateurs, ils ont tendance à être plus vastes.

Il n'y a donc pas de politique d'éducation nationale en Allemagne - et c'est pourquoi les médias importants se sont, pendant longtemps, assez peu intéressés aux sujets touchant à l'école. Après tout, pourquoi un journal ou bien une radio suprarégionale devrait-elle couvrir des événements tels que le lancement d'une expérimentation scolaire dans tel Land ou encore les résultats particulièrement mauvais au baccalauréat dans tel autre - hormis, bien sûr, le fait que la plupart des Länder n'ont de toute façon pas de structure centralisée pour le baccalauréat, les sujets d'examen dépendant des établissements et des enseignants.

L'école a ainsi longtemps été l'affaire de l'échelon régional, provincial. Ce qui fait une bonne école, ce qu'est un bon professeur, ce que les enfants devraient apprendre - rien de tout cela ne présentait d'intérêt pour les médias. Ils auraient tout aussi bien pu publier sur les associations de jardins ouvriers !

\section{LA SOCIÉTÉ SE MÉFIE dE L’ÉCOLE}

À cela s'ajoute un deuxième aspect important concernant l'école en Allemagne: son rôle est perçu de manière très ambivalente par les médias également. Alors que les parents français confient, au plus tard dès trois ans, leurs enfants toute la journée aux enseignants, on considère en Allemagne l'école comme un lieu désagréable, dont il faut protéger les enfants le plus longtemps possible - une conséquence de notre vision romantique de la famille, en particulier de la mère : elle est le principal éducateur des enfants. L'enseignant n'est pas responsable de l'éducation mais seulement de l'apprentissage de la littératie et de la numératie ${ }^{4}$.

Diverses raisons ont conduit l'Allemagne à maintenir la demi-journée d'école. Mais cette particularité a des conséquences importantes sur le rôle de l'école, sur la réputation et l'image des enseignants dans les médias. L'école n'est pas, contrairement à de nombreux pays, le lieu de vie principal des élèves. Ils rentrent chez eux à midi, où leur mère, qui travaille au plus à mi-temps, les

4. « Mach Dich locker Mama » (« Détends-toi maman »), Süddeutsche Zeitung, 8-9 mai 2004. 
accueille avec un repas. Les après-midi sont alors consacrés - en fonction des intérêts et du milieu social - aux jeux vidéo, à regarder la télé, à des activités sportives dans des clubs, au piano, à la danse ou aux leçons d'équitation. La vraie vie se déroule donc en dehors de l'école.

Il s'ensuit que l'enseignant est considéré comme quelqu'un qui ne doit travailler qu'à mi-temps - le temps nécessaire à la préparation des cours et aux corrections étant volontiers négligé. Ajoutez à cela les congés foisonnants. Dans l'ensemble, beaucoup d'Allemands ont l'impression que les enseignants ont un emploi à mi-temps confortable, tout en étant payés à plein temps et en bénéficiant de plus de congés que les cinq à six semaines habituelles. Les enseignants eux-mêmes contribuent au fait qu'ils sont considérés comme des fonctionnaires à mi-temps, car presque toutes les associations professionnelles sont farouchement opposées à toute tentative d'introduire des cours l'après-midi, de rémunération à la performance ou - ce qui ne surprendra pas - de suppression du statut de fonctionnaire.

S'ajoute à cela une érosion générale du rôle de l'autorité, conséquence du mouvement des années 1968. L'enseignant, tout comme le prêtre et le médecin, a vu son influence décliner. L'expansion de l'éducation dans les années 1970, qui a été associée à la construction de nombreux nouveaux lycées et à des besoins d'enseignants plus grands, a également altéré le prestige de la formation des enseignants. L'opinion publique a une mauvaise image de l'enseignant et les médias contribuent à perpétuer ce point de vue. Lorsque Gerhard Schröder, qui deviendra plus tard chancelier, était encore premier ministre de Basse-Saxe, il qualifia les enseignants de «types paresseux ${ }^{5}$ », provocant la colère de nombreux enseignants, mais exprimant dans l'ensemble une opinion courante ${ }^{6}$.

\section{L'ÉCOLE, UN NON SUJET POUR LES MÉDIAS}

Deux aspects essentiels caractérisent donc la couverture médiatique de l'école dans le dernier quart du $\mathrm{XX}^{\mathrm{e}}$ siècle : une image tendanciellement négative des enseignants et un débat idéologique sur la structure de l'école. On aurait pu penser que le débat politique sur la «bonne » structure scolaire aurait trouvé un écho dans les médias. Cela n'a pas été le cas, en tout cas dans les années quatrevingt et quatre-vingt-dix. La phase d'expansion de l'éducation était passée. Dans les années soixante-dix, un certain optimisme régnait encore, on croyait à "la promotion par l'éducation ». Mais les deux décennies suivantes ont apporté un certain désenchantement.

Parmi l'ensemble des sujets politiques, l'école est considérée comme une question seulement régionale, à laquelle les médias ne s'intéressent qu'au sein 
d'un Land. Un journal suprarégional ou une chaîne de télé ne consacreront pas plus d'une page hebdomadaire ou d'un magazine aux questions scolaires, car cela ne fait gagner ni lecteur ni part d'audience. Les réunions régulières des ministres de l'éducation sont soit ignorées, soit tournées en ridicule - le chancelier Helmut Kohl qualifiait la Conférence permanente des ministres de l'éducation de "tortue ", du fait de l'immobilisme de ces assemblées ministérielles.

\section{LE CHOC PISA}

Avec les résultats de l'enquête internationale PISA (Programme international pour le suivi des acquis des élèves), qui ont traversé en 2001 l'Allemagne comme une onde de choc, la situation a pourtant évolué. Plus précisément, un débat avait déjà débuté au milieu des années quatre-vingt-dix, tout d'abord sous l'égide des chercheurs en éducation, puis parmi les responsables des politiques éducatives. En 1995, l'Allemagne participe pour la première fois à une enquête internationale, l'enquête TIMMS (Trends in International Mathematics and Science Study). Les mauvais résultats obtenus par l'Allemagne éveillent l'intérêt politique et public pour le thème de l'école. La conférence des ministres de l'éducation avait décidé, en 1997, que les écoles allemandes devaient participer à des tests internationaux pour garantir la qualité de l'enseignement ${ }^{7}$. Pour la première fois, l'école était considérée comme une responsabilité nationale plutôt que comme une question locale relevant des seize Länder. Politique et société commencent à s'intéresser à l'école. Les médias traditionnels comme le Süddeutsche Zeitung, le Frankfurter Allgemeine Zeitung, ou Die Zeit élargissent leur couverture médiatique de ces sujets. Si, pendant des décennies, les questions scolaires n'ont qu'occasionnellement trouvé leur place dans les actualités, des pages thématiques hebdomadaires sont désormais inaugurées.

Avec la publication des résultats de PISA (l'Allemagne se situe dans la moyenne inférieure - au dessous de la moyenne de l'OCDE) l'intérêt public grandit à nouveau et, avec lui, la couverture médiatique ${ }^{8}$. Pour mémoire, 2001 est également l'année de la fin de l'essor sans précédent des start-up et de l'éclatement de la bulle spéculative sur les valeurs technologiques. L'Allemagne a ainsi vécu de près la mondialisation. Une conséquence de cette mondialisation est la confrontation des systèmes éducatifs et la possibilité d'un débat public sur des questions telles que : comment traitons-nous les enfants issus de l'immigration, qui quittent plus souvent que les autres l'école sans diplôme? Les bacheliers allemands sont-ils trop âgés ? Le Gymnasium devrait-il ne durer que huit ans,

7. Anne Overesch, „Wie die Schulpolitik ihre Probleme (nicht) löst: Deutschland und Finnland im Vergleich“, Waxmann Verlag, 2007 (Comment la politique de l'école (ne) résout (pas) ses problèmes : comparaison entre l'Allemagne et la Finlande).

8. «Die PISA-Analyse: Sind deutsche Schüler doof? », Der Spiegel, 13 décembre 2001 (L'analyse de PISA : les élèves allemands sont-ils bêtes ?). 
au lieu des neuf années actuelles? Les étudiants doivent-ils finir l'université plus tôt pour pouvoir rivaliser avec les titulaires de licence français et de Bachelor britanniques?

En comparaison avec d'autres pays, l'écho suscité en Allemagne par la première enquête PISA est considérable. Une analyse de l'OCDE montre que, sur la période allant de décembre 2001 à janvier 2002, plus de 600 articles de presse ont été publiés en Allemagne, bien plus que dans tous les autres pays ayant participé à PISA. En Angleterre et en Autriche, l'OCDE a comptabilisé environ 120 articles de journaux, 25 en France et 30 aux États-Unis. Même si les Allemands font preuve d'un penchant avéré pour l'autocritique, cela atteste en fin de compte d'un véritable intérêt public. Les journalistes en éducation se rendent dans les pays scandinaves, en particulier en Finlande, pour comprendre comment les pays nordiques parviennent à obtenir ces bons résultats, en particulier en matière de justice sociale. Les Scandinaves sont considérés, en général, comme les champions de PISA, même si certains pays asiatiques ont fait mieux. Les systèmes éducatifs sud-coréens ou japonais demeurent suspects aux yeux des Allemands. Ces pays sont soupçonnés de faire peser inutilement sur leurs élèves une pression à la performance - et donc on leur préfère l'exemple scandinave ${ }^{9}$.

Mais l'école est en Allemagne une compétence des Länder. Un an et demi après le choc PISA, un second choc frappe l'Allemagne, du moins certains Länder allemands. Alors que les résultats Land par Land sont rendus publics, il apparaît que les différences entre Länder sont très importantes. En gros, les Länder gouvernés par les partis conservateurs (Bavière, Bade-Wurtemberg, Thuringe, Saxe) obtiennent de meilleurs résultats que ceux traditionnellement gouvernés par les sociaux-démocrates (Rhénanie du Nord-Westphalie, Brême, Berlin). De nouveau, le débat public s'exacerbe et la couverture médiatique s'accroît.

\section{PISA, UN PHÉNOMÈNE MÉdIATIQUE}

Dans l'ensemble, les années 2000 et 2001 ont marqué un tournant dans le traitement de la question de l'école par les médias. PISA est devenue une somme de tous les problèmes du système scolaire allemand - et ce, indépendamment du fait que l'enquête porte dans les faits sur les résultats des élèves âgés de 15 à 16 ans : l'âge au moment de l'entrée à l'école, la pression aux résultats dans les écoles primaires de certains Länder, où les élèves sont réorientés en fonction de leurs notes après la quatrième année, l'enseignement frontal fréquent, les enseignants vieillissants.

9. «Das finnische Erfolgsgeheimnis », Die Zeit, 2 avril 2008 (" Les secrets du succès finlandais »). (NdT) 
Le fait que l'Allemagne réagisse si vivement aux résultats de PISA est un exemple classique d'un phénomène médiatique d'entraînement politique et sociétal. Les ministres de l'éducation, en prenant la décision de participer à des tests internationaux, en avaient jeté les bases, le grand public ressentant déjà un certain malaise face à des problèmes scolaires évidents. Les médias se sont alors emparés du sujet, déclenchant à nouveau une publicité qui a accru la pression sur le gouvernement.

L'émotion suscitée par les résultats de PISA est également visible à travers les émissions de télévision et les livres qui ont repris le thème pour luimême, sans véritablement en rendre compte. Par exemple, la télévision publique a diffusé une série d'émissions sur PISA, inspirées de jeux télévisés à succès. L'émission " PISA - le test des Länder. À quel point l'Allemagne est-elle bête ? », lancée en octobre 2003, a été suivie par huit millions de téléspectateurs. Après le test des Länder, un test des pays et un test de vacances - toujours sous l'appellation «PISA » ont suivi.

De nombreux livres ont surfé sur la vague PISA : La grande formation PISA pour les adultes. Tester, développer et appliquer des connaissances intelligentes ou encore Cours accéléré PISA pour les adultes. 600 exercices d'entraînement niveau débutant, moyen et avancé sont des ouvrages spécialisés plutôt courants. Par ailleurs, certaines offres suggèrent aux parents de préparer leurs enfants à PISA, et donc à l'école et à la vie, comme par exemple : "Mon enfant est-il prêt pour PISA ? ». Le terme «PISA » devient synonyme d'astuce, d'intelligence et de compétence.

\section{De LA JUSTICE SOCIALE À LA PRESSION SCOLAIRE : LES ARGUMENTS DES ACTEURS}

Il va sans dire que les médias ont des tonalités différentes. Les médias dits conservateurs vont par exemple se concentrer sur les bons résultats d'un Land comme la Bavière, doté d'un système scolaire segmenté ; les médias de centregauche sur la question sociale en particulier, autour des raisons qui expliquent pourquoi, en Allemagne, l'appartenance sociale des parents a un tel impact sur la réussite scolaire. La fixation sur une vision idéologique du système éducatif, qui déterminait déjà le débat auparavant, subsiste. Elle est principalement propagée par les partis et les syndicats d'enseignants, qui sont les principaux informateurs des médias. Ceux-ci relaient volontiers des interprétations à l'emporte-pièce.

Les médias ont volontiers recours aux arguments des principaux acteurs du débat. Outre les responsables des politiques éducatives, il s'agit des syndicats d'enseignants, ainsi que des organisations d'employeurs, traditionnellement fortes en Allemagne. Les parents jouent un rôle plus mineur dans le débat public, car leurs intérêts sont très diffus et il n'existe pas d'association nationale de parents d'élèves qui ait du poids. Les conseils d'élèves restent également des acteurs mineurs. 
Les points de vue des employeurs sont relativement homogènes : ils voient en PISA une confirmation de leur posture traditionnelle, qui considère que l'éducation des élèves est médiocre et que leur volonté ne cesse de se relâcher. Ils utilisent dès lors le débat pour renforcer et marteler leur position. Les associations, ainsi que certaines grandes entreprises, proposent alors des services stratégiques qui s'occupent d'éducation. Sur la plupart des questions, comme par exemple la journée entière d'école, les syndicats et les entreprises se rejoignent - même s'ils poursuivent des intérêts différents : l'entreprise souhaite avant tout pour que les femmes soient disponibles comme force de travail, les syndicats recherchent la justice sociale. Depuis PISA, les employeurs sont d'ailleurs les plus ardents défenseurs de la journée entière d'école et soulèvent très régulièrement la question dans le débat public. Ce sont également eux qui réclament que les élèves soient plus sollicités et fournissent plus d'efforts; mais également que les enseignants soient mieux formés et payés à la performance. De manière générale, ils appellent à une nouvelle culture de l'effort ${ }^{10}$.

Les syndicats défendent des positions plus différenciées, à partir desquelles les médias réagissent en fonction de leurs propres logiques. Le principal syndicat d'éducation de gauche, membre de la puissante Fédération des syndicats allemands, est le Syndicat de l'éducation et des sciences (Gewerkschaft Erziehung und Wissenschaft - GEW). Pour cet acteur, le problème principal réside dans les mauvais résultats obtenus par les Länder gouvernés par les sociaux-démocrates, ce que l'organisation explique globalement par la structure socioéconomique de ces Länder (en gros, les Länder gouvernés par le SPD sont ceux où les revenus sont les plus faibles). Le syndicat considère de manière générale les tests de performance avec scepticisme - un bon mot apprécié du GEW est : «Ce n'est pas en le pesant que le cochon s'engraisse ». Pourtant, le GEW utilise l'enquête PISA pour renforcer sa position et légitimer ses objectifs : les résultats sont pour lui avant tout la preuve qu'en Allemagne, l'accès à l'éducation est fortement tributaire de l'origine sociale. Il appelle à abolir le système différencié et à accompagner les élèves de manière plus individualisée, en particulier ceux issus de l'immigration.

Les organisations d'enseignants, traditionnellement conservatrices, se positionnent à l'opposé de cela, et en particulier l'Association des philologues allemands, qui représente les professeurs de lycée. Ils considèrent également avec un certain scepticisme les tests de performance internationaux, mais, à l'inverse du GEW, ils n'ont pas de problème de principe avec ces tests. Ils doutent davantage de la comparabilité des résultats de différents systèmes scolaires et critiquent le fait que les exercices de PISA soient conçus selon les programmes et méthodes d'apprentissage anglo-saxons. Néanmoins, ils utilisent PISA pour réaffirmer leurs

10. «Mehr Kooperation, Expertenempfehlungen für neue Impulse in der deutschen Bildungspolitik " (« Plus de coopération, recommandations d'experts pour une nouvelle impulsion de la politique éducative allemande "), Fondation des Télécommunications allemandes, Bonn, 2012. 
objectifs : un tronc commun d'études obligatoire, avec en particulier plus de cours d'allemand, une meilleure orientation des élèves dans les écoles différenciées - l'exact opposé de la position du GEW.

\section{UNE NOUVELLE ÈRE DE L'INFORMATION}

Il existe cependant un consensus assez répandu pour dire que la politique d'éducation régionale allemande a conduit à des débats idéologiques qui ont assez duré. Le titre d'un article dans l'hebdomadaire Die Zeit résume cela très bien : "Tester au lieu de croire ${ }^{11}$. On contrôle désormais les élèves à défaut d'être convaincu d'avoir le bon système éducatif. Dans l'ensemble, les médias sont également d'accord pour dire que l'Allemagne a négligé le débat public sur l'école beaucoup trop longtemps, et que les médias n'ont pas fourni une couverture appropriée. C'est la conséquence positive des mauvais résultats de PISA : l'éducation n'est plus une question abstraite, elle est devenue tangible.

Mais l'agitation médiatique retombe vite. À chaque nouvelle enquête PISA, la couverture médiatique diminue. Cela n'est pas surprenant : les événements récurrents sont en général moins captivants que ce qui est inattendu. De plus, les élèves allemands ont amélioré leurs résultats à PISA au fil des ans. Ainsi, la possibilité du scandale diminue également. La dernière enquête, qui mettait l'accent sur la résolution de problèmes et qui a été rendue publique en avril 2014, n'a donc à nouveau pas réellement fait la une. Faute de pouvoir dénoncer la mauvaise performance des élèves allemands, les journalistes doivent se concentrer sur les détails de l'étude, comme par exemple les raisons pour lesquelles les garçons réussissent moins bien en lecture que les filles.

La politique éducative a un problème fondamental : elle joue sur de nombreux leviers, la formation des enseignants, la taille des classes, la structure scolaire, les programmes. Et ce qui est problématique, c'est que, bien souvent, on ignore totalement l'influence de ces différents paramètres. Par exemple, la plupart des parents sont convaincus que leurs enfants apprennent mieux dans des classes plus petites. Mais sur ce sujet, il n'existe pas une seule étude qui montre que - en dehors de très petits groupes - la taille de classe a un effet positif sur l'apprentissage. Le seul facteur véritablement décisif est la qualité de l'enseignement. Mais il est très difficile de mesurer à quel point un enseignant transmet bien ou mal la matière qu'il enseigne, et cela n'est pratiquement pas contrôlé.

Pour aggraver les choses, les succès des politiques éducatives s'observent à long terme. Par exemple, si les professeurs de mathématiques étaient mieux formés, cela n'aurait d'effet qu'après trois, quatre ou cinq années au plus tôt, lorsque les enseignants entrent pour la première fois dans une salle de classe.

11. « Testen statt glauben », Die Zeit, 18 mai 2000. 
Mais la politique et les médias veulent du court terme et c'est pourquoi, d'une part, les réformes nécessaires ne sont pas souvent mises en œuvre et, d'autre part, les médias ne s'y intéressent généralement pas.

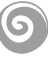

En résumé, l'enquête internationale PISA a fait évoluer l'opinion publique allemande sur les questions scolaires, ainsi que la politique éducative. Notamment, PISA a permis que la politique éducative cesse de se cantonner à la perspective régionale. Par conséquent, la couverture médiatique sur l'éducation s'est renforcée. Les questions scolaires sont maintenant considérées comme dignes d'intérêt et les médias se sont adaptés à cet intérêt, et l'ont même attisé. La politique doit également s'adapter à ce phénomène. Si les questions scolaires ne font toujours pas gagner une élection, elles peuvent en revanche les faire perdre - comme cela a été le cas avec le parti au pouvoir en Bavière, la CSU, qui a perdu, en 2008, sa majorité absolue, entre autres raisons parce que les parents étaient mécontents du raccourcissement de la durée du lycée ${ }^{12}$.

Combien de temps, cependant, durera cet intérêt nouveau des Allemands pour les politiques éducatives ? On ne peut que spéculer à ce sujet. Peut-être le déclin du taux de natalité permettra-t-il à l'éducation et à la formation de voir leur valeur rehaussée, et aux questions scolaires de continuer à jouer un rôle de premier plan dans le débat public et les médias. Il n'est cependant pas exclu, après la phase de forte croissance des questions éducatives dans les médias, que l'on atteigne un seuil de saturation et que l'intérêt retombe à nouveau.

12. "Quo vadis CSU ? Die bayerische Landtagswahl 2008 und ihre Folgen » : www.kas.de/upload/dokumente/.../ Volksparteien_strohmeier.pdf 
